\title{
Assessment of Optimal Bragg Grating Length for Optical performance using Two Apodization Functions
}

\author{
Dr. Jabbar Kh. Mohammed \\ Communication Engineering Departement, University of Technology \\ drjab68@gmail.com,11021@uotechnology.edu.iq
}

\begin{abstract}
Fiber Bragg gratings (FBGs) are one of the most effective technologies because of their suitability in many applications of the fiber techniques. Moreover, it can be utilized in sensing elements and estimating the physical parameters of the optical fiber. In the current research, various fiber bragg lengths are considered and a comparative investagtion is made for the performance of the refractive index modulation at two different apodization profiles namely: Gaussian index profiles and uniform index profiles. Also, a comparative study of the optical communication system performance has been carried out of refractive indexes with two apodization functions. A comprehensive comparison in terms of gain, noise figure, OSNR, quality factor, bit error rate and power has been performed where the performance of the optical system is studied under various optical channel lengths (from $25 \mathrm{~km}$ to $100 \mathrm{~km}$ ) with various FBG lengths, which are ranged from $4 \mathrm{~mm}$ to $14 \mathrm{~mm}$ in both apodization profiles, i.e. uniform profile and Gaussian profile. In order to carry out the simulation of the performance RZ, the optisystem version 9 is used for single channel based on over single mode fiber. The results showed that the maximum Q.F. as the performance parameter is obtained at the FBG length of $8 \mathrm{~mm}$ at all optical lengths. Moreover, the varation effect of optical fiber channel length was greater than the varation effect of refractive index for all the studied parameters (gain, noise figure, OSNR, quality factor, BER, and power) for two apodization functions.
\end{abstract}

Index Terms - Apodized FBG, Single mode optical Fiber, Q-Factor, Optsisystem

\section{INTRODUCTION}

Over the past decades, fiber optics have come a long way from their evolution, with new technology creating new applications, in turn, supplying money to develop more new technologies. In the late 1990s, the growth increased as the Internet fed a seemingly limitless thirst for a bandwidth that is only provided by optical fibers [1].

With advances in technology, the fiber optics has become the backbone of the global telecommunications network, giving us instant access to telephones and web sites around the world. When we use a cell phone, the calls usually go wireless only to the tower, where a fiber-optic cable is connected to the telephone networks. The demand for bandwidth continues to rise, although there's a lot of surplus fiber in the ground right now. Fiber revolutionized telecommunications just as the railroads revolutionized transportation in the nineteenth century. The new technology used fiber optic that has a high speed data rate and very wide capacity compared with the conventional electrical communication. The main systems of the optical communication consist of the transmitter, which converts and transmits an electronic signal into a light signal, the receiver, which converts light into electricity by using a photoelectric effect, and the optical channels, which are used for transmitting optical bit streams by optical fibers [2] [3]. 
The apodization technique is one of the most important techniques, which is used in many applications of fiber bragg gratings, where it deals with optimizing sensor performances by using anodized FBG [4]. In this work, a comprehensive studies were introduced on the impact of using a different apodization profile Uniforms, and Gaussian on the performances of FBG. The performance evaluation parameters, which were tested in this study, are Fiber Bragg grating, to get the results in gain, signal to noise ratio, output power, noise figure, bit error rate, and Q. Factor with varouse fiber bragg grating lengths.

\section{THE APODIZATION OF FIBER BRAGG GRATING IN OPTICAL FIBER}

The advantages of the Fiber Bragg Grating (FBG) are simple construction, high wavelength discrimination, polarization sensitivity, low loss due to the insertion, and full compatibility with universal single mode of the optical fibers communication system [5]. The fiber bragg grating is a kind of the distributed bragg reflectors which is structured in a short segment for the optical fiber, which can reflect specific wavelengths of transmits and light all others [6], where FBG can reflect a thin spectral segment of the light which is directed into the core of the optical fiber at the Bragg wave-length, which in turn depends on the optical flber refractive index and the period of the fiber grating. FBG can be defined as one mode through presenting the simple periodic form of the powerful UV, which leads to increase the permanence and exposure of the refractive index [7], where the fixed index grating is generated due to exposure pattern formation. Once the light source is provided into the FBG, there is only a small range of light wavelengths matching to the Bragg wavelength, which in turn is reflected transferring the spectral data to the involved FBG spectrum analyzer. While other wave-lengths partly reflect at small index variants and delay destructively, which leads to transmit those wavelengths. Figure 1 shows the basics of the Bragg's light [8].

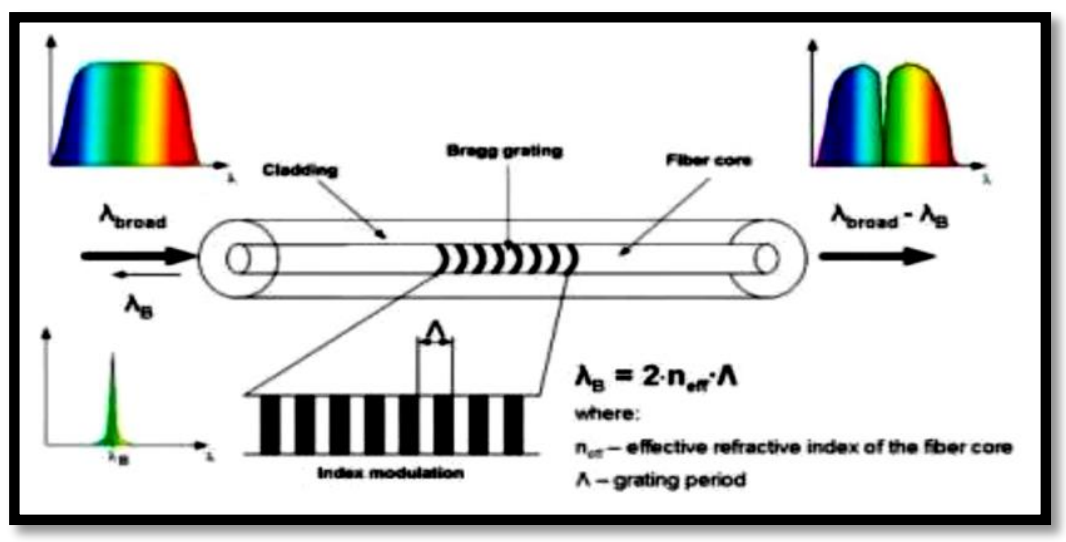

Figure 1 The Fiber Bragg Gratting

The apodization can be defined based on the grating spectral response by uniform index modulation beside of the fiber length which has harmonic on the side of the main part as undesirable. Another definition of the apodization is the variant of the the modulation distance alongside the grating length. FBG plays a major role in the elimination of the side lobes in order to keep reflectivity and create a thin bandwidth. The grating spectral is fourier transform of the envelope of index modulations with grating. The Gaussian apodization is a method of suppressing side lobes to apodize the index profile such that towards the edges of the grating the index modulation approaches to zero. In the simple case, when a fixed-length grid has a uniform shape of a constant refractive index, the main peak or bragg ring is accompanied by a series of lateral lobes at adjacent wavelength. In the WDM application, which requires significant rejection of adjacent channels, it is important to reduce the reflectivity of lateral lobes, or to apodize the grating reflection spectrum. The direct using of a natural Gaussian intensity with expanded laser beam allows for the exposition of Gaussian apodized Bragg gratings [9][10]. In the current work, two types of the apodization profiles are investigated, namely Uniform and Gaussian Apodized cases. 
If a uniform FBG is formed within the core of an optical fiber with an average refractive index $n_{0}$, then the refractive index can be expressed as [10][11]:

$$
n=n_{o}+\Delta n(z) \cos \left(\frac{2 \pi}{\Lambda} z\right)=\text { constant }
$$

And the wavelength of the Bragg is denoted by:

$$
\lambda_{B}=2 n_{e f f} \Lambda
$$

where $\mathrm{z}$ represents the distance along the FBG longitudinal axis, $\Delta \mathrm{n}$ represents the amplitude of the induced refractive index, the grating spacing is denoted by $\Lambda$, and $n_{\text {eff }}$ is the effective grating index. The Gaussian Apodized Case is given by:

$$
f(z)=e^{\left\{-4 \log (2)\left[\frac{z-L / 2}{S . L}\right]^{2}\right\}}
$$

Where $\mathrm{L}$ is the grating length and $\mathrm{Z}$ is the light propagation coordinate lengthwise the FBG length, respectively. S represents the taper variable utilized with a smooth tuning of the reflection spectrum [12].

\section{THE SETUP OF SYSTEM SIMULATION}

The optical communication system (OCS) includes three components namely: receiver, transmitter and transmission channel, where the receiver comprises a photodetector. The transmister includes three parts which are pseudo random bit sequence generator, wave laser $(\mathrm{CW})$, modulator and pseudo random bit sequence generator. For the transmission channel, a Single-mode Optical fiber was utilized with a length up to $100 \mathrm{~km}$. The attenuation coefficient of the transmission channel was $0.2 \mathrm{~dB} / \mathrm{km}$. In the simulation setup, the input signal was produced through coming back to zero pseudo-random binary-sequence. In Mach-Zehnder modulator, the input signal was controlled using wave laser type of continuous, where the input signal was delivered using wave length of continuous laser of $1550 \mathrm{~nm}$. Moreover, the power was greatly modified at $20 \mathrm{Gbps}$ at vrious modulation arrangements in the Mach-Zehnder modulator. The extinction ratio of $25 \mathrm{~dB}$ was employed. In the current OCS FGB, the refractive index type of uniform profile and Gaussian profile was investigated, where the refractive index was varied between 1.45 and 1.48 .

The EDFA (Erbium-doped fiber amplifier) was utilized in order to control the signal amplification and quality factor compensation. In EDFA, the signal was amplified before the reception by a photo detector PIN and then the amplified signal was passed into a Bessel optic filter, where the Bessel optical filter has a bandwidth of $40 \mathrm{GHZ}$. The optisystem software diagram of the designed and simulated model of the modulator is shown in figure 2 .

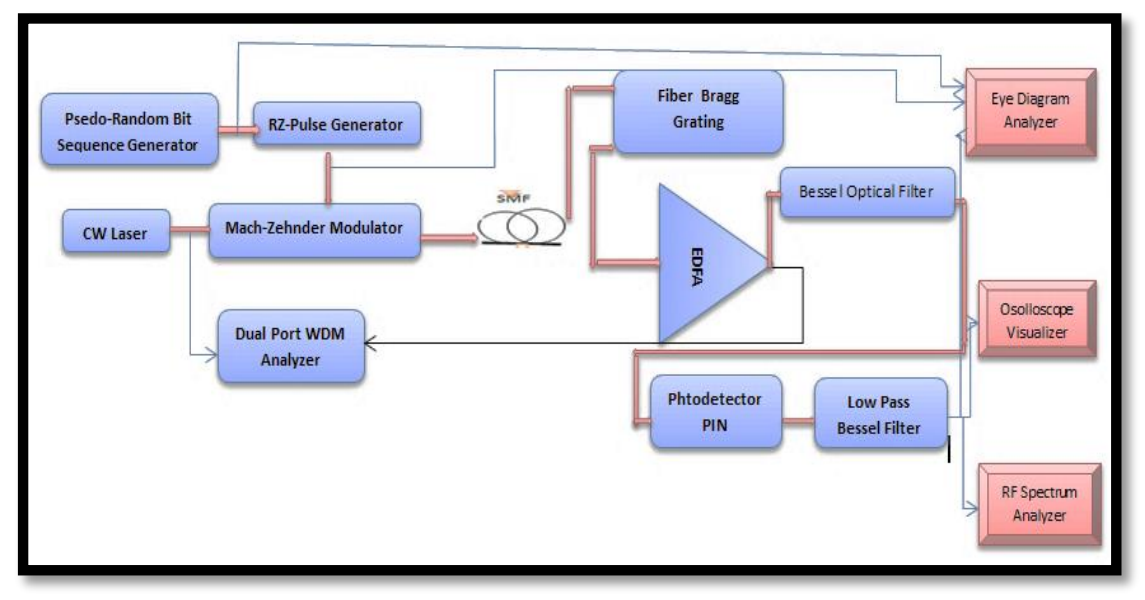

FIGURE 2. OPTISYSTEM SOFTWARE DIAGRAM OF THE DESIGNED AND SIMULATED MODEL OF THE MODULATOR 


\section{THE DISCUSSION OF SIMULATION RESULTS}

In this research, Opti-system software is used to carry out the simulations and parametric studies for the performance of the optical fiber transmission system in the format of FBG based on two apodization functions. In the current study, the pefromance of two types from apodization functions namely: Uniform and Gaussian have been investigated. Furthermore, comprehensive comparisons have been conduted in terms of gain, noise figure, Q-factor, OSNR, BER, and the average output power in order to deliver the benefits and drawbacks of optical communications system for various apodization FBG profiles.

Figure 3 (a) and (b) show six fiber bragg grating lengths that are investigated along the optical channel length to obtain the optimal fiber bragg length at maximum Q.F for both cases in terms of uniform and Gaussian profiles. In figure 3(a), based on uniform apodazation, the optimum value of the Q.F. at FBG length of $8 \mathrm{~mm}$ is obtained comperd with othe five lengths. It can be seen from figure 3(b) that the best performance in terms of Q.F. can be obtained at an FBG length of $10 \mathrm{~mm}$ for the range from $4 \mathrm{~mm}$ to $14 \mathrm{~mm}$.
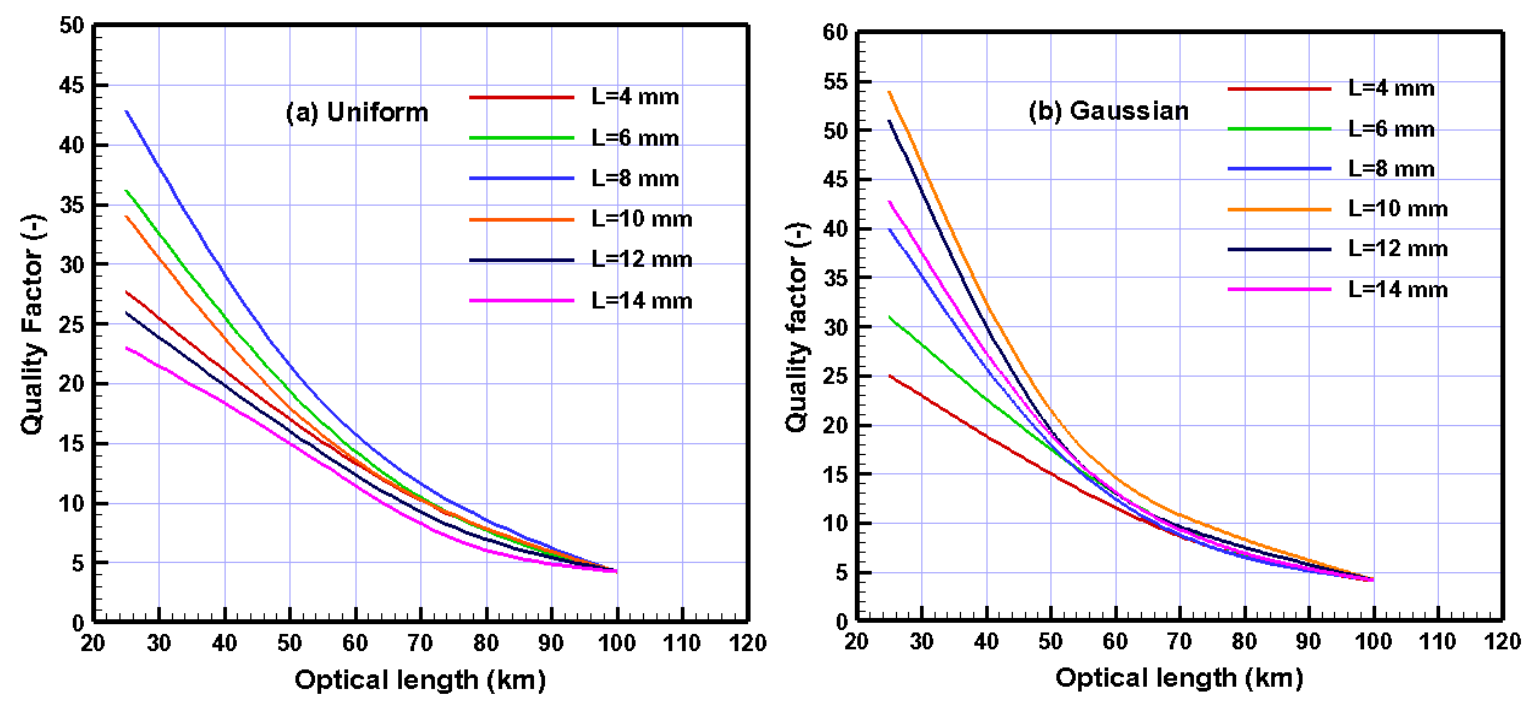

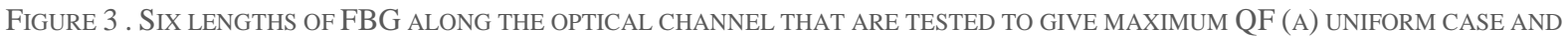
(B) GAussian CASE

Figure 4 shows the relation between Q-factor and out power with varation of FBG lengths for two apodization forms in terms of uniform and Gaussian at a channel length of $50 \mathrm{~km}$ and a refractive index of 1.47. With a maximum Q-factor of 21.5 for both cases, the FBG length for the uniform is $8 \mathrm{~mm}$, but for the Gaussian case, we must increase the FBG length to $10 \mathrm{~mm}$ to give the same value of Q-factor of 21.5. This means that more cost and more complexity for the same performance compared to uniform apodization. So that the output power (right scale of figure) for uniform is better than Gaussian along FBG length, and the o/p power of uniform at $8 \mathrm{~mm}$ is better than the o/p power of Gaussian at 10 and $8 \mathrm{~mm}$. Figure 5 shows the gain, noise figure and OSNR of both cases with varation of FBG lengths, where all performances for uniform in $8 \mathrm{~mm}$ are better than Gaussian in $8 \mathrm{~mm}$ or $10 \mathrm{~mm}$. The difference is very clear between the two cases, therefore $8 \mathrm{~mm}$ of FBG is a better length than the other length.

The association of the refractive indexes for the uniform and Gaussian profiles at the fiber Bragg grating of $8 \mathrm{~mm}$ and optical channel length of $50 \mathrm{~km}$ is shown in Figures 6 and 7.

It can be ssen from the comparisons of Figures 6 and 7 that all ratings for the apodization FBG refractive index were ranged from 1.45 to 1.48 with a step of 0.01 . The single fiber connection distance was fixed at $50 \mathrm{~km}$. The results for uniform profiles cases at a refractive index of 1.47 showed that the Q-factor, gain, noise figure, and output power are $21.22,12.59 \mathrm{~dB}, 17.9 \mathrm{~dB}$ and $16.58 \mathrm{dBm}$, respectively. On the 
other hand, the Gaussian profile results showed that the Q-factor, gain, noise figure, and output power are $20.57,12.15 \mathrm{~dB}, 20.6 \mathrm{~dB}$, and $16.18 \mathrm{dBm}$, respectively. As can be noted, all parameters namely: noise figure, Gain, Q-factor and output power increase with increasing the fiber refractive index. Moreover, the results revealed that the uniform profile case deliverd good results compared with those of the Gaussian profile.

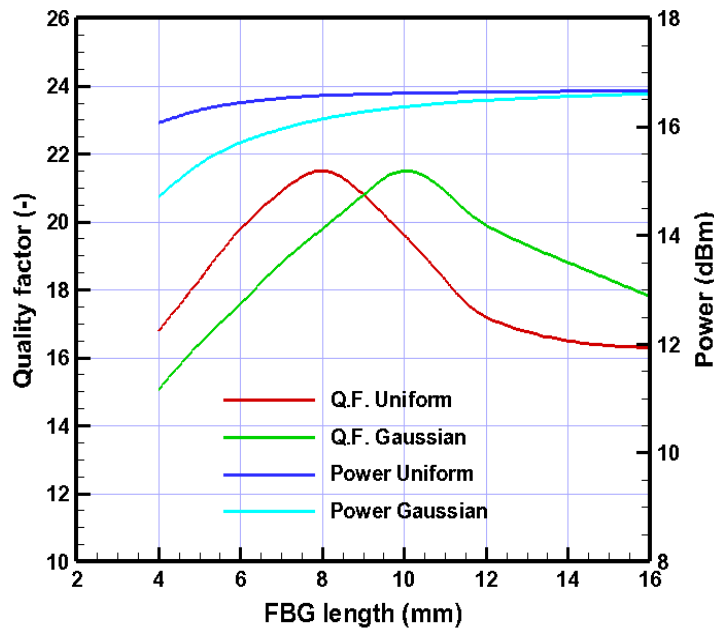

FIGURE 4. QUALITY FACTOR AND O/P POWER FOR UNIFORM AND GAUSSIAN CASES

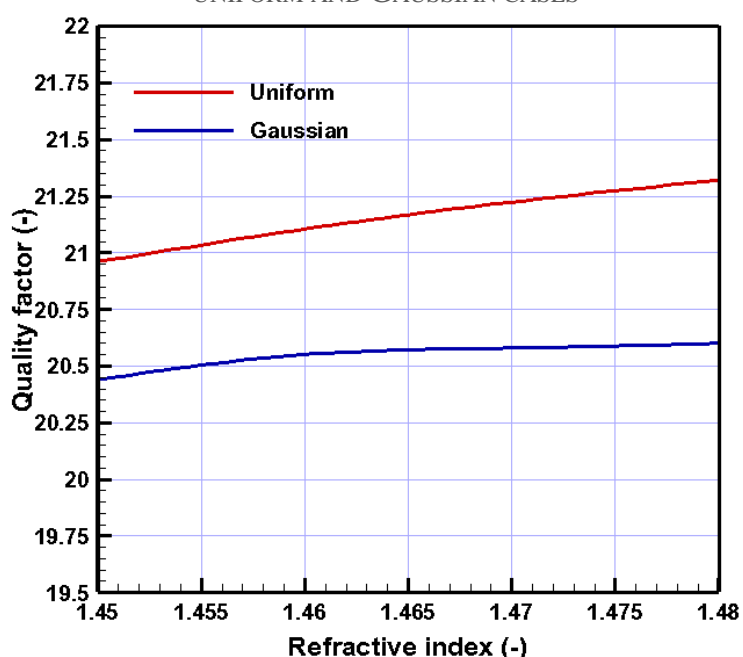

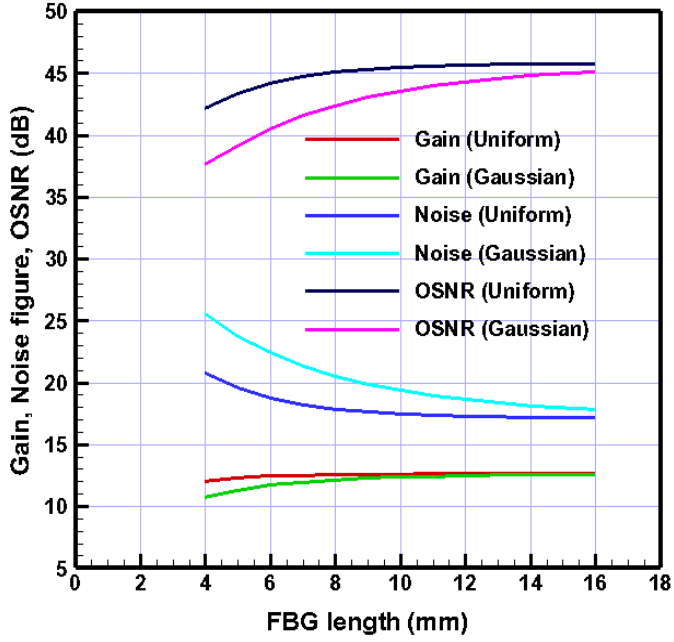

FIGURE 5. GAIN, NOISE FIGURE AND OSNR FOR UNIFORM AND GAUSSIAN CASES

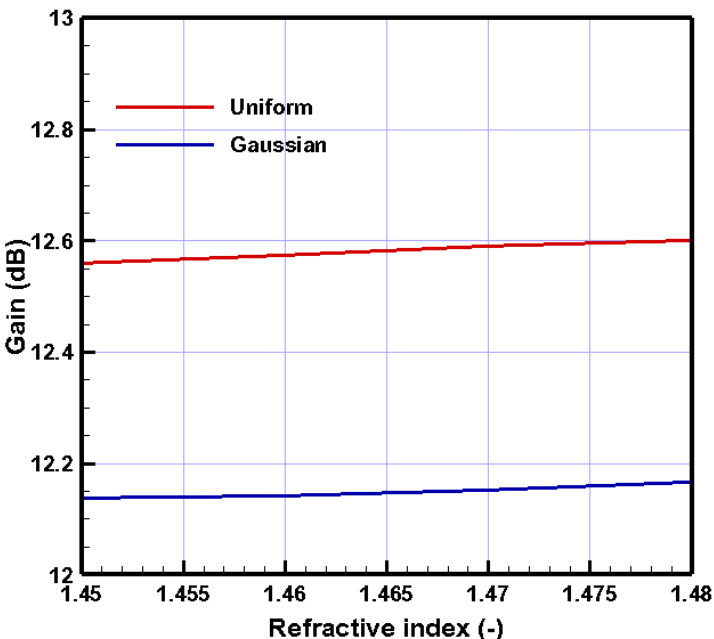

FIGURE 6 REFRACTIVE INDEX FOR TWO CASES OF THE APODIZATION (A) WITH Q. FACTORS, (B) WITH GAIN
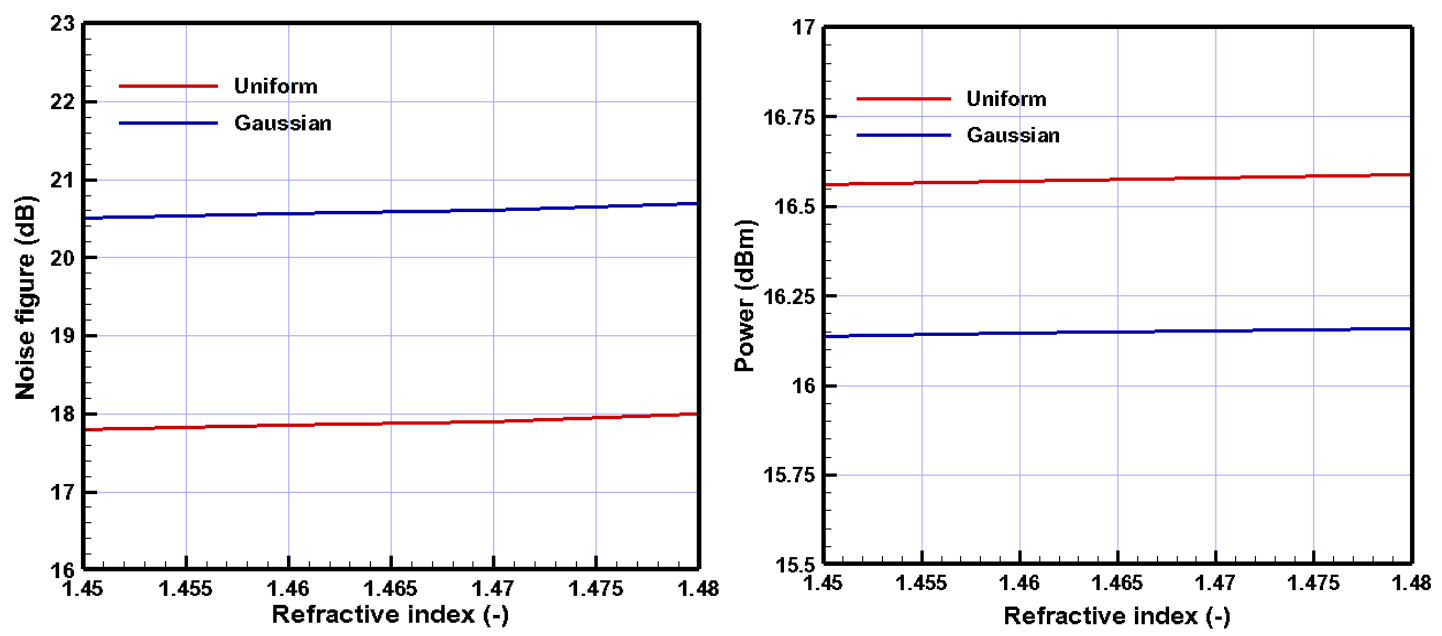

FIGURE 7 REFRACTIVE INDEX FOR TWO CASES OF THE APODIZATION (A) WITH N.F. , (B) WITH OUTPUT POWER 
Figure 8 shows minimum bit error rate at an optimal grating length of $(8 \mathrm{~mm})$ and $50 \mathrm{~km}$ optical fiber channel for two apodization techniques along refractive index from 1.45 to 1.48 , where it can be seen that increasing the refractive index improves the bit error rate for both cases, and the performance with uniform apodization is better than that of the Gaussian apodization for all values of the refractive index.

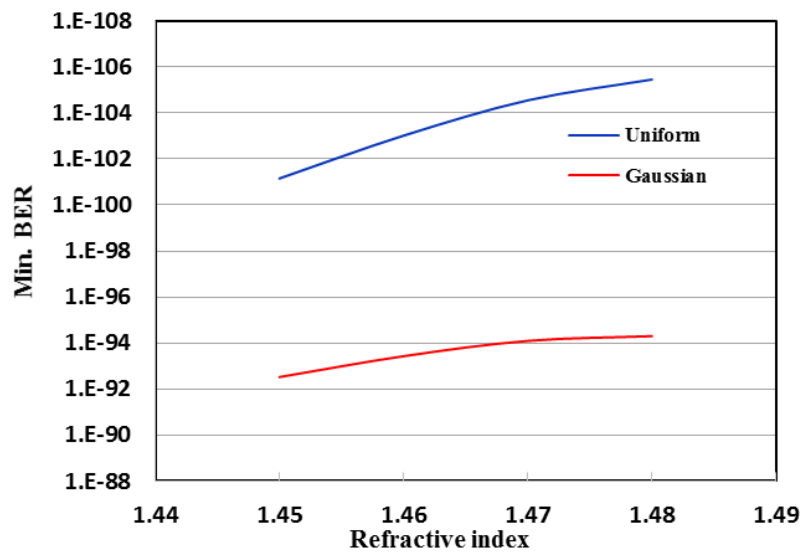

Figure 8. PERFormance OF Min. BER WITH REFRACTIVE INDEX FOR UNIFORM AND GAUSSIAN CASES

Figure 9 (left scale) indicates the change of the Q. factor with optical fiber length at two profiles, namely uniform and Gaussian. As can be noted, the maximum value of the Q. factor based on uniform profile was 21.5 compared to 20 with Gaussian profiles at the optical fiber length of $50 \mathrm{~km}$. Moreover, the variation of the Q.F. decreases until the length reached about $100 \mathrm{~km}$.

Figure 9 (right scale) shows the change in output power with the optical fiber length at two different profiles namely: uniform and Gaussian. The maximum output power of $16.68(\mathrm{dBm})$ was noticed at a length of $50 \mathrm{~km}$ based on the uniform profile compared with $16.19(\mathrm{dBm})$ using the Gaussian profile. The deviation in the output power with these two profiles increases with increasing the optical fiber length. Also, the output power is decreasing with increasing the optical fiber length, where at $100 \mathrm{~km}$, the minimum output power with the uniform profile was $13.5(\mathrm{dBm})$ compared to $12.1(\mathrm{dBm})$ with the Gaussian profile.

Figure 10 shows the variation of the gain, noise figure and output signal to noise ratio (OSNR) with the optical fiber length for both uniform and Gaussian profiles. It can be seen from Figure 10 that the gain based on the uniform profile is better than that of the Gaussian profile along the optical fiber length, where the maximum difference between these two profiles at the optical length of $50 \mathrm{~km}$ was $0.6 \mathrm{~dB}$ compared to $1 \mathrm{~dB}$ at the length of $100 \mathrm{~km}$. The variation in noise figure with the optical fiber length at uniform and Gaussian profiles is shown in Figure 10. As one can see, the variation was almost linear and the noise figure based on the Gaussian profile has higher values compared with the uniform profile, where the maximum value of the N.F. with the Gaussian profile was $20.5 \mathrm{db}$ compared with $17.7 \mathrm{db}$ using the uniform profile at $50 \mathrm{~km}$. It can be concluded from Figure 10 that the noise figure with the uniform profile is better than that of the Gaussian profile along the length, so that the OSNR for the uniform is better than that of the Gaussian apodization function along the distance.

Figure 11 shows the minimum bit error rate at the optimal grating $(8 \mathrm{~mm})$ and other length $(4 \mathrm{~mm})$ along the optical fiber channel for two apodization techniques; the value of the minimum BER at $50 \mathrm{~km}$ with optimal FBG length of $8 \mathrm{~mm}$ was 2.88E-105 and 4.8E-63 with FBG length of $4 \mathrm{~mm}$ for the uniform case. With the Gaussian case at the same distance, the minimum BER with optimal FBG length of $8 \mathrm{~mm}$ was 1.9E-94 and 1.2E-51 with FBG length of $4 \mathrm{~mm}$, where the optimal length of fiber bragg grating led to improve the performance compared with the other length. The BER performance with the uniform is better than that of the Gaussian case along the optical length.

Figure 12 shows the variation of FBG length with the optical fiber length based on the maximum vlues of Q.F. Therefore, the optimal FBG length can be calculated at any distance of the optical channel, such 
as for $50 \mathrm{~km}$ channel length with a maximum value of Q.F., which led to an optimal FBG length at 8 $\mathrm{mm}$ for the uniform case and $10 \mathrm{~mm}$ for the Gaussian case.

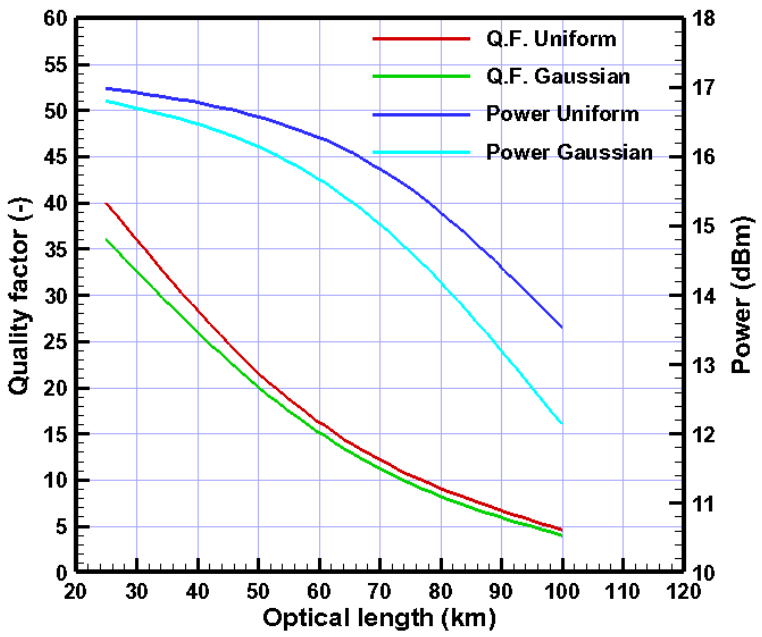

FIGURE 9. Q.F AND O/P POWER FOR UNIFORM WITH 8 MM FBG LENGTH

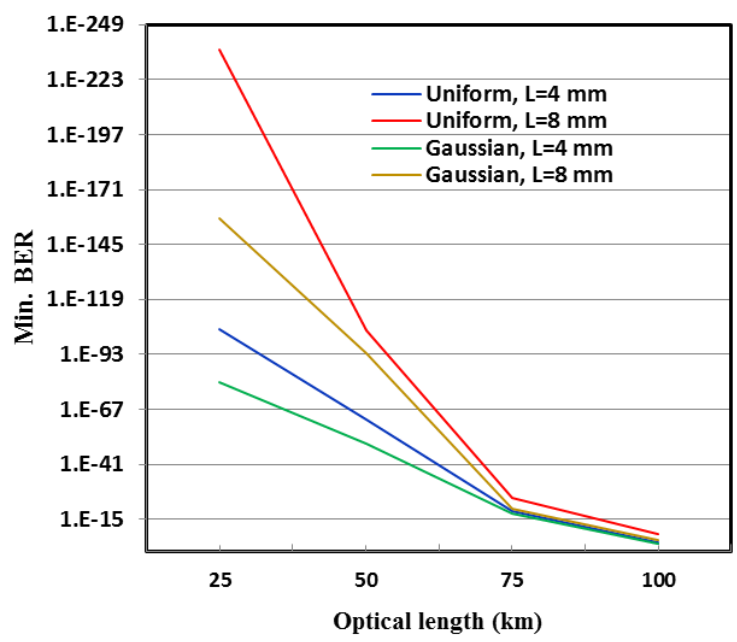

Figure 11 THE VARIATION OF THE MIN.BER WITH THE OPTICAL FIBER AT 8 MM FBG LENGTH

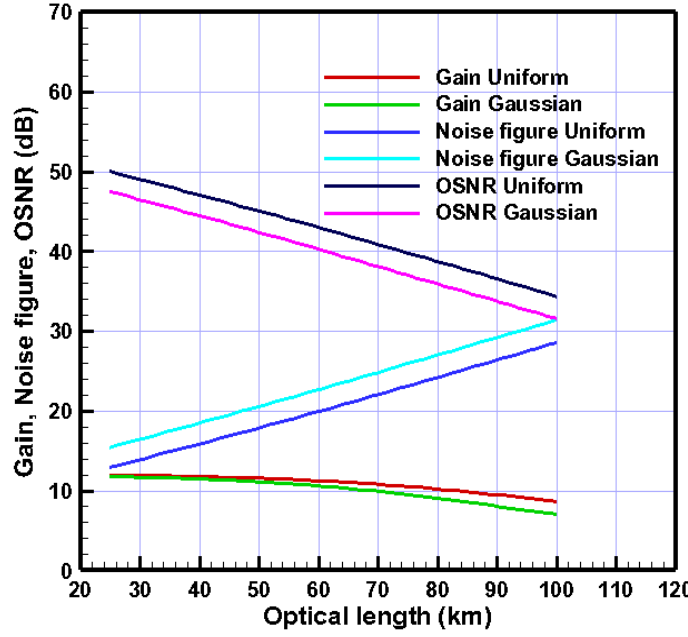

FIGURE 10. GAIN, N.F AND OSNR FOR UNIFORM AND GAUSSIAN CASES AND GAUSSIAN CASES WITH 8 MM FBG LENGTH

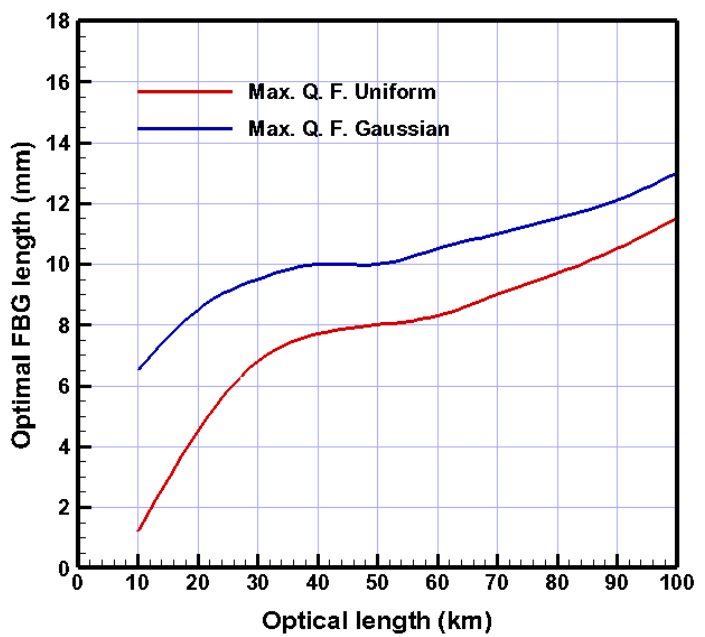

Figure 12 The OPTIMAL FBG LENGTH WITH EACH POINT OF THE OPTICAL FIBER LENGTH

\section{CONCLUSION}

In the present work, the performance evaluation of the fiber Bragg gratings (FBG) has been investigated with two cases; uniform and Gaussian, where various apodization profiles have been offered.

The results indicated that the increasing of the refractive index (at an optical fiber length of $50 \mathrm{~km}$ and $8 \mathrm{~mm}$ FBG length) leads to improve performance by increasing Q.F and the linear increase of other investigated parameters namely; noise figure, gain, BER, OSNR and output power. The uniform profile at a higher refractive index leads to high quality factor and gain with a low bit error rate than that of the Gaussian case in the fiber bragg grating optical communication system.

The effect of the FBG length with the optical channel length for both apodization profiles, i.e. uniform profile and Gaussian profile, has been investigated, where the quality factor as the optical system performance parameter is examined. The best value of the Q.F. was obtained at an FBG length of $8 \mathrm{~mm}$ with the uniform apodization profile along the optical channel length. The increase in the optical fiber length at a fixed refreactive of 1.47 with $8 \mathrm{~mm}$ FBG length led to increase the noise figure, and BER 
comparing with deacresing the gain, Q.F., OSNR and the output power. As a result, the comparison between both studies for both cases in terms of refractive index and optical fiber length showed that the variation effect of the optical fiber length at 1.47 refractive index on the parameters (i.e. gain, Q.F., noise factor, BER, and output power) was greater than the variation in the refractive index with $50 \mathrm{~km}$ channel length, and the optimal length of FBG of $8 \mathrm{~mm}$ led to improve the performance of the optical system compared with other lengths.

\section{REFERENCES}

[1] Deepak Sh., Payal and Rajbir S. "Performance Investigation of Dispersion Compensation Techniques in 32-Channel DWDM System" International Journal of Electronics, Electrical and Computational System IJEECS ,Volume 6, Issue 11 November 2017

[2] John A." Fundamentals of Optical Fibers", Second Edition,2004

[3] Govind P. A., "Fiber Optic Comm. Systems", Third Edition, John Wiley \& Sons, Inc., New York, 2002

[4] Patrick H.J.Kersey, A.D., Davis, M.A., , LeBlanc, M., Koo, K.P., M.A. and Friebele, E.Askins, C.G., Putnam, J., "Fiber grating sensors," IEEE Journal of Lightwave Technology, 15(8), 1442-1463, (1997).

[5] Kenneth O. Hill and Gerald M. "Fiber Bragg Grating Technology Fundamentals and Overview", Journal of Lightwave Technology, Vol. 15, No. 8, August 1997

[6] Mishra, Ramchandra; Bharti, Ramesh "An Analysis of 10 Gbits/S Optical Transmission System Using Fiber Bragg Grating (FBG) and Doube EDFA". International Journal of Advanced Research in Computer Science . Sep/Oct 2017, Vol. 8 Issue 8, p349-355.

[7] Ayad Z. Mohammed, A. K. Abass, Samar K. Ibrahim, Wail Y. Nassir. Theoretical Analysis of Fiber Bragg Grating Tunable Filter Utilizing Tensile /Compression Technique. Diyala Journal of Engineering Sciences, Vol. 11, No. 2, June 2018, pages 55-59

[8] JYOTSNA R. M., MANISHA CH.," Spectral Characteristic of Uniform Fiber Bragg Grating Using Couole Mode Theory", International Journal of Electrical, Electronics and Data Communication, ISSN (p): 2320-2084, Volume-1, Issue-, July-2013

[9] M. A. Mirza, PhD, S. M. Bilal, PhD Scholar; M. Zafrullah, PhD. " simulation and analysis of Gaussian apodized fiber bragg grating strain sensor," University of Engineering and Technology, Taxila ; 47050, Pakistan. , 2012

[10] Ali Mahdi Hammadi, Ali Nasser Hussain "Performance Analysis of Apodized Fiber Bragg Gratings Formats in Optical Communication System" The International Journal Of Engineering And Science, Volume 5, Issue 10 , PP 40-45, 2016

[11] Kumar S., Khare A. ,Singh J. "Design of Gaussian apodized fiber Bragg grating and its applications “ International Journal of Engineering Science and Technology". 2010. V 2. No 5. P. 1419-1424.

[12] I. Ashry A. Elrashidi2 ,A. Mahros3, M. Alhaddad, and K. Elleithy ,'Investigating the Performance of Apodized Fiber Bragg Gratings for Sensing Applications “ Conference of the American Society for Engineering Education , Proceedings of 2014 